\title{
Article
}

\section{THE BAR PATTERN SPEED OF DWARF GALAXY NGC 44311}

Corsini, E M, Aguerri, J A L, Debattista, Victor P, Pizzella, A, Barazza, F D and Jerjen, $\mathrm{H}$

Available at https://clok.uclan.ac.uk/16859/

Corsini, E M, Aguerri, J A L, Debattista, Victor P orcid iconORCID: 0000-00017902-0116, Pizzella, A, Barazza, F D and Jerjen, H (2007) THE BAR PATTERN SPEED OF DWARF GALAXY NGC 44311. The Astrophysical Journal, 659 . L121L124. ISSN 0004-637X

It is advisable to refer to the publisher's version if you intend to cite from the work. http://dx.doi.org/10.1086/518035

For more information about UCLan's research in this area go to http://www.uclan.ac.uk/researchgroups/ and search for <name of research Group>.

For information about Research generally at UCLan please go to http://www.uclan.ac.uk/research/

All outputs in CLoK are protected by Intellectual Property Rights law, including Copyright law. Copyright, IPR and Moral Rights for the works on this site are retained by the individual authors and/or other copyright owners. Terms and conditions for use of this material are defined in the policies page.

\section{CLoK}

Central Lancashire online Knowledge www.clok.uclan.ac.uk

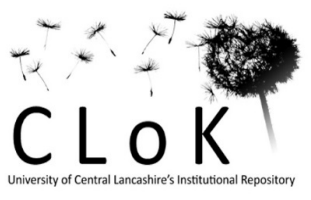




\title{
THE BAR PATTERN SPEED OF DWARF GALAXY NGC $4431^{1}$
}

\author{
E. M. Corsini, ${ }^{2}$ J. A. L. Aguerri, ${ }^{3}$ Victor P. Debattista, ${ }^{4,5}$ A. Pizzella, ${ }^{2}$ F. D. Barazza, ${ }^{6}$ And H. Jerjen $^{7}$ \\ Received 2007 February 13; accepted 2007 March 13; published 2007 March 27
}

\begin{abstract}
We present surface photometry and stellar kinematics of NGC 4431, a barred dwarf galaxy in the Virgo Cluster undergoing a tidal interaction with one of its neighbors, NGC 4436. We measured its bar pattern speed using the Tremaine-Weinberg method and derived the ratio of the corotation radius, $D_{L}$, to the bar semimajor axis, $a_{B}$. We found $D_{L} / a_{B}=0.6_{-0.4}^{+1.2}$ at $99 \%$ confidence level. Albeit with large uncertainty, the probability that the bar ends close to its corotation radius (i.e., $1.0 \leq D_{L} / a_{B} \leq 1.4$ ) is about twice as likely as that the bar is much shorter than the corotation radius (i.e., $D_{L} / a_{B}>1.4$ ).
\end{abstract}

Subject headings: galaxies: elliptical and lenticular, cD — galaxies: individual (NGC 4431) galaxies: kinematics and dynamics — galaxies: photometry — galaxies: structure

\section{INTRODUCTION}

Stellar bars are observed in a substantial fraction of nearby disk galaxies (Knapen et al. 2000; Eskridge et al. 2000; Marinova \& Jogee 2007). Their growth is partly regulated by the exchange of angular momentum with the stellar disk and the dark matter (DM) halo. For this reason the dynamical evolution of bars can be used to constrain the distribution of DM in the inner regions of galaxy disks. Using perturbation theory, Weinberg (1985) predicted that a bar would lose angular momentum to a massive DM halo through dynamical friction, slowing down in the process. This prediction was subsequently confirmed in $N$-body simulations (Debattista \& Sellwood 1998, 2000; Athanassoula 2003; O’Neill \& Dubinski 2003; Sellwood $\&$ Debattista 2006). They found that bars are slowed efficiently within a few rotation periods if a substantial density of DM is present in the region of the bar. On the other hand, if the mass distribution is dominated by the stellar disk throughout the inner few disk scale lengths, then the bar remains rapidly rotating (here defined as the bar ending just inside its corotation radius) for a long time. Thus, the accurate measurement of the bar pattern speed, $\Omega_{p}$, provides a way to discriminate whether the central regions of the host galaxy are dominated by baryons or by DM.

Bar pattern speeds are most usefully parameterized by the distance-independent ratio $\mathcal{R}=D_{L} / a_{B}$, where $D_{L}$ is the corotation radius and $a_{B}$ the semimajor axis of the bar. Bars that end near corotation $(1 \leq \mathcal{R} \leq 1.4)$ are termed fast, while shorter bars $(\mathcal{R}>1.4)$ are said to be slow. The best way to determine the bar rotation parameter $\mathcal{R}$ is by measuring $\Omega_{p}$ with the model-independent method developed by Tremaine \& Weinberg (1984, hereafter TW84). To date, this method has been used to measure $\Omega_{p}$ of mainly massive $\left(V_{c} \gtrsim 150 \mathrm{~km} \mathrm{~s}^{-1}\right)$, early-

\footnotetext{
${ }^{1}$ Based on observations collected at the European Southern Observatory, Chile [ESO programs 71.B-0138(A) and 73.B-0091(A)].

${ }^{2}$ Dipartimento di Astronomia, Università di Padova, I-35122 Padova, Italy; enricomaria.corsini@unipd.it, alessandro.pizzella@unipd.it.

${ }^{3}$ Instituto de Astrofísica de Canarias, E-38200 La Laguna, Spain; jalfonso@iac.es.

${ }^{4}$ Astronomy Department, University of Washington, Seattle, WA 98195 1580; debattis@astro.washington.edu.

${ }^{5}$ Brooks Prize Fellow.

${ }^{6}$ Department of Astronomy, University of Texas at Austin, Austin, TX 78712-0259; barazza@ astro.as.utexas.edu.

${ }^{7}$ Research School of Astronomy and Astrophysics, Mount Stromlo Observatory, Australian National University, Weston ACT 2611, Australia; jerjen@ mso.anu.edu.au.
}

type barred galaxies (see Corsini 2004 and references therein), and it has been successfully tested against $N$-body simulations (Debattista 2003; O’Neill \& Dubinski 2003). All measured bars are compatible with being fast, implying that the inner regions of these galaxies are not dominated by DM. The only exception appears to be the slow bar in NGC 2915, a blue compact dwarf galaxy, for which the TW84 method was applied to H I radio synthesis data (Bureau et al. 1999).

The minimum possible amount of DM in disk galaxies can be determined from the rotation curve, by scaling up the massto-light ratios of the luminous components to fit the central velocity gradient (see Bosma 1999 for a review). However, the lack of any obvious transition region from the bulge/diskdominated to the halo-dominated part of rotation curves severely limits the ability to obtain a unique decomposition and derive the central DM distribution (e.g., Corsini et al. 1999). The structure of DM halos is more directly revealed in galaxies where the luminous components are thought to give a nearly zero contribution to the mass budget, because this makes mass modeling easier and the derived DM distribution less uncertain. For this reason dwarf galaxies were considered ideal targets for studying the properties of DM halos and for testing if they have a central cuspy power-law mass density distribution as predicted by cold dark matter (CDM) cosmology (Navarro et al. 1996, 1997; Moore et al. 1998, 1999). However, recent extensive studies have shown that in most of them the contribution of the stellar disk can account for the inner rotation curve, and DM halos with a constant-density core provide a better fit to than those with a density cusp (e.g., de Blok \& Bosma 2002; Swaters et al. 2003; Spekkens et al. 2005; Gentile et al. 2005).

Nonetheless, the interpretation of these results has been controversial because of possible observational systematic effects, such as the poor spatial resolution of radio maps (van den Bosch \& Swaters 2001) and slit misplacement in optical spectroscopy (Swaters et al. 2003), as well as noncircular (Hayashi \& Navarro 2006), and off-plane gas motions (Swaters et al. 2003; Valenzuela et al. 2007).

Since measurements of pattern speeds with the TW84 method do not require high spatial resolution or slits passing exactly through the galaxy center and refers to the stellar component only, such a measurement in a dwarf disk galaxy can test unambiguously for the presence of a dense DM cusp. In the present Letter we attempt such a measurement for NGC 4431. 


\section{NGC 4431}

NGC 4431 (VCC 1010$)$ is a small $\left(1.7^{\prime} \times 1.1^{\prime}\right.$; de Vaucouleurs et al. 1991, hereafter RC3) and faint $\left(B_{T}=13.74\right.$; RC3 $)$ nucleated dwarf galaxy (Binggeli et al. 1985) classified as dSB0/a by Barazza et al. (2002), following an unsharp mask analysis of the galaxy. The presence of a bar had earlier been missed by virtue of the galaxy's low luminosity and the relatively small angle between the bar's and disk's position angle (P.A.). Both the presence of a strong bar and trailing arms (which are clearly visible in Fig. 3 of Barazza et al. 2002) and the rotationally supported kinematics (Simien \& Prugniel 2002; Pedraz et al. 2002) are indicative of a genuine disk galaxy. The total absolute magnitude of the galaxy is $M_{B_{T}}^{0}=-17.41$ corrected for inclination and extinction (RC3) and adopting a distance of $16.2 \mathrm{Mpc}$ (Jerjen et al. 2004). It is located close to the center of the Virgo Cluster at a projected distance of $\sim 300 \mathrm{kpc}$ from M87. Its closest neighbor in projection is the dE6/dS0 galaxy NGC 4436, at a projected separation of $3.7^{\prime}$ or $\sim 18 \mathrm{kpc}$.

\section{BROADBAND IMAGING}

Deep broadband imaging of NGC 4431 was carried out with the Very Large Telescope (VLT) at the European Southern Observatory on 2000 April 1, as part of a project aimed at measuring distances, metallicities, and ages of dwarf galaxies in the Virgo Cluster. Details of observations, data reduction, and analysis are reported elsewhere (Barazza et al. 2003; Jerjen et al. 2004).

We analyzed the $R$-band image of NGC 4431 obtained by Barazza et al. (2003), with foreground stars and background galaxies subtracted. We fitted ellipses to galaxy isophotes with the $\operatorname{IRAF}^{8}$ task ELLIPSE, after masking the northeastern part of the image to minimize the light contamination from NGC 4436, the outskirts of which fall in the field of view of the image. We first fitted ellipses allowing their centers to vary to test whether patchy dust obscuration and tidal deformations were present. Having found no evidence of varying ellipse centers within the errors of the fits for the inner $40^{\prime \prime}$, we concluded that in these regions there is little or uniform obscuration and no tidal deformations, and that the galaxy is a viable candidate for the TW84 method. At larger radii, the shift of ellipse centers $\left(\approx 1^{\prime \prime}\right)$ of NGC 4431 in the direction of NGC 4436 may be due to the interaction between the two galaxies. The ellipse fits were then repeated with the ellipse center fixed, and the resulting surface brightness profile is plotted in Figure $1 d$. The inclination $\left(i=48.7^{\circ} \pm\right.$ $1.5^{\circ}$ ) and disk P.A. (P.A. .disk $=6.9^{\circ} \pm 1.0^{\circ}$ ) were determined by averaging the values measured between $50^{\prime \prime}$ and $70^{\prime \prime}$. They are consistent within the errors with values obtained by fitting ellipses with free centers.

We measured $a_{B}$ (Fig. 1) using four independent methods based on Fourier amplitudes (Aguerri et al. 2000), Fourier and ellipse phases (Debattista et al. 2002), and a parametric decomposition of the surface brightness profile (Prieto et al. 2001). The mean of the resulting values is our best estimate of $a_{B}$, and we assume the largest deviation from the mean as our error estimate $\left(a_{B}=21.9^{\prime \prime} \pm 1.5^{\prime \prime}\right)$. The bar length is in agreement with the radius where the spiral arms seen in the unsharp mask image of Barazza et al. (2002) start.

\footnotetext{
${ }^{8}$ IRAF is distributed by NOAO, which is operated by AURA, Inc., under contract with the National Science Foundation.
}

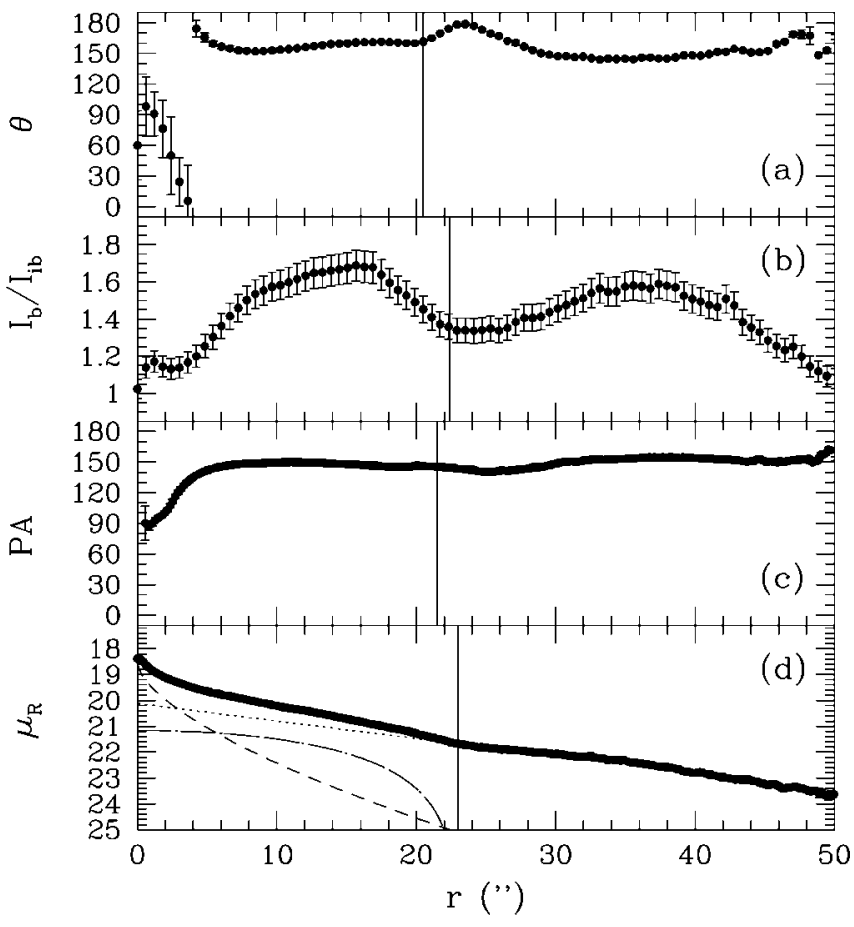

FIG. 1.-Bar semimajor axis length of NGC 4431. (a) Phase angle of $m=2$ Fourier component. (b) Bar/interbar intensity ratio. (c) P.A. of the deprojected isophotal ellipses. (d) Surface brightness decomposition in bulge (dashed line), disk (dotted line), and bar (dash-dotted line). The vertical lines show the value of $a_{B}$ obtained with each model.

\section{LONG-SLIT SPECTROSCOPY}

The spectroscopic observations of NGC 4431 were carried out in service mode at VLT on 2003 May 4-6 (run 1), 2004 April 21-22 (run 2), and 2004 May 18-23 (run 3). The Focal Reducer Low Dispersion Spectrograph 2 (FORS2) mounted the volume-phased holographic grism GRIS_1400V+18 with 1400 grooves $\mathrm{mm}^{-1}$ and the $0.7^{\prime \prime} \times 6.8^{\prime}$ slit. The detector was a mosaic of two MIT/LL CCDs. Each CCD had $2048 \times 2068$ pixels of $15 \times 15 \mu \mathrm{m}^{2}$. The wavelength range from 4560 to $5860 \AA$ was covered with a reciprocal dispersion of $0.645 \AA$ pixel $^{-1}$ and a spatial scale of $0.250^{\prime \prime}$ pixel $^{-1}$ after a $2 \times 2$ pixel binning. We obtained spectra with the slit at P.A. $=7.9^{\circ}$ crossing the galaxy center $(3 \times 45$ minutes in run 1$)$ and offset by $5.0^{\prime \prime}$ eastward $(4 \times 30$ minutes in run 3$)$. We also obtained spectra with the slit at P.A. $=6.9^{\circ}$ and shifted by $5.0^{\prime \prime}$ eastward $(2 \times 40$ minutes in run 2;2 $\times 40$ minutes in run 3) and westward $(4 \times 40$ minutes in run 3) with respect to the galaxy nucleus. Two different P.A.'s were chosen for the slits to test for the sensitivity of the TW84 measurement to the $\sim 1^{\circ}$ uncertainty in P.A. disk . Comparison lamp exposures obtained for each observing night ensured accurate wavelength calibrations. Spectra of G and K giant stars served as kinematical templates. The average seeing FWHM was $1.2^{\prime \prime}$ in run $1,0.8^{\prime \prime}$ in run 2 , and $0.9^{\prime \prime}$ in run 3 . Using standard MIDAS ${ }^{9}$ routines, all the spectra were bias-subtracted, flat-field corrected, cleaned of cosmic rays, corrected for bad pixels, and wavelength-calibrated as in Debattista et al. (2002). The accuracy of the wavelength rebinning $\left(\approx 1 \mathrm{~km} \mathrm{~s}^{-1}\right)$ was checked by measuring wavelengths of the brightest night-sky emission lines. The instrumental resolution was $1.45 \pm 0.01 \AA$ (FWHM) corresponding to $\sigma_{\text {inst }}=35 \mathrm{~km} \mathrm{~s}^{-1}$ at $5170 \AA$. The spectra obtained in the same run along the same position were co-added

${ }^{9}$ MIDAS is developed and maintained by the European Southern Observatory. 


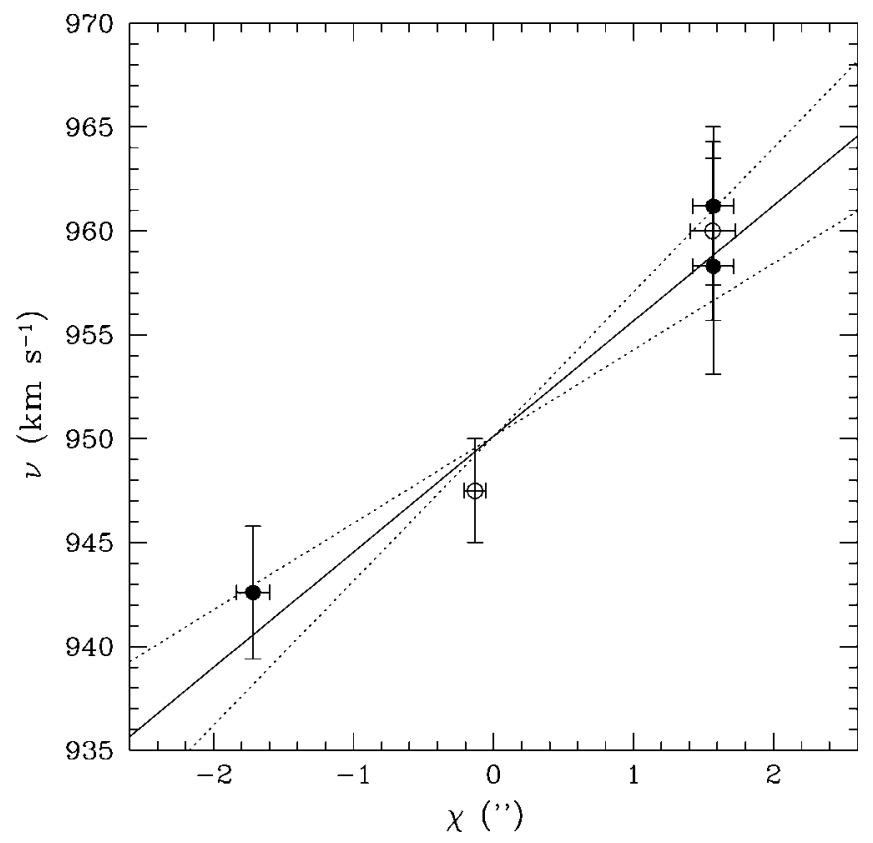

FIG. 2.-Pattern speed of the bar in NGC 4431. The kinematic integrals $\mathcal{V}$ are plotted as a function of the photometric integrals $\mathcal{X}$. The best-fitting straight line has a slope $\Omega_{p} \sin i=5.56 \pm 1.39 \mathrm{~km} \mathrm{~s}^{-1} \operatorname{arcsec}^{-1}$. Open circles correspond to slits at P.A. $=7.9^{\circ}\left(Y=0^{\prime \prime}\right.$ and $\left.Y=+5.0^{\prime \prime}\right)$, while filled circles are for slits at P.A. $=6.9^{\circ}\left(Y= \pm 5.0^{\prime \prime}\right)$.

using the center of the stellar continuum as reference. In the resulting spectra the sky contribution was determined by interpolating along the outermost $\approx 30^{\prime \prime}$ at the edges of the slit and then subtracted.

\section{PATTERN SPEED OF THE BAR}

To measure the pattern speed of the bar, $\Omega_{p}$, we used the TW84 method. For slits parallel to the disk major axis, this relates the luminosity-weighted mean position, $\mathcal{X}$, to the luminosity-weighted mean velocity $\mathcal{V}=\mathcal{X} \Omega_{p} \sin i$, where $i$ is the galaxy inclination.

To measure $\mathcal{V}$ for each slit, we first collapsed the twodimensional spectrum along its spatial direction between $-90^{\prime \prime}$ and $+90^{\prime \prime}$ in the wavelength range between 5220 and $5540 \AA$, obtaining a one-dimensional spectrum. The value of $\mathcal{V}$ was then derived by fitting the resulting spectrum with the convolution of the spectrum of the G0 III star SAO 119458 and a Gaussian line-of-sight velocity profile by means of the Fourier correlation quotient (FCQ) method (Bender 1990) as done in Aguerri et al. (2003, hereafter ADC03). We estimated uncertainties by Monte Carlo simulations with photon, readout, and sky noise. As a precision check we analyzed separately the two eastward-offset spectra $\left(Y=+5.0^{\prime \prime}\right)$ that were obtained with the slit at P.A. $=6.9^{\circ}$ in runs 2 and 3 , respectively. The two resulting estimates of $\mathcal{V}$ are in agreement within the errors (Fig. 2). To compute $\mathcal{X}$ for each slit, we extracted the luminosity profile from the $R$-band image along the position of the slit after convolving the image to the seeing of the spectrum. The $R$-band profile matches very well the profile obtained by collapsing the spectrum along the wavelength direction, supporting that the slit was placed as intended. We used the $R$-band profiles to compute $\mathcal{X}$ because they are less noisy than those extracted from the spectra, particularly at large radii. By comparing the slits at P.A. $=6.9^{\circ}$ and at P.A. $=7.9^{\circ}$ we also verified that the error introduced by the $\sim 1^{\circ}$ uncertainty in disk

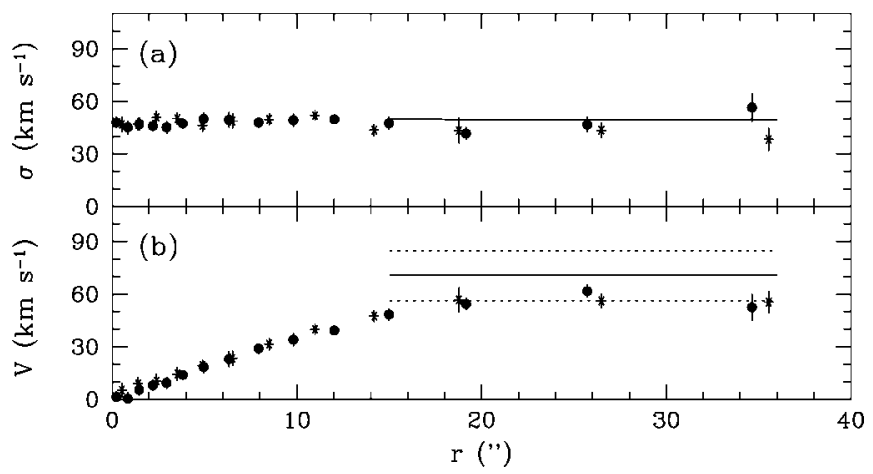

FIG. 3--(a) Major-axis radial profile of the stellar line-of-sight velocity dispersion fitted with an exponential profile at $r \geq 15^{\prime \prime}$ (solid line). (b) Majoraxis radial profile of the stellar line-of-sight velocity (after subtracting the systemic velocity $V_{\mathrm{sys}}=948 \pm 3 \mathrm{~km} \mathrm{~s}^{-1}$ ) and the $V_{c} \sin i$ curve (solid line) with errors (dotted lines) obtained by applying the asymmetric drift for $r \geq$ $15^{\prime \prime}$ as in ADC03. In (a) and $(b)$ the measured profiles are folded around the center, with filled circles and asterisks referring to the north (receding) and south (approaching) sides, respectively.

P.A. is small for both $\mathcal{X}$ and $\mathcal{V}$. We obtained $\Omega_{p}$ by fitting a straight line to the values of $\mathcal{X}$ and $\mathcal{V}$ to the data for slits at $Y= \pm 5.0^{\prime \prime}$ and P.A. $=6.9^{\circ}$ and at $Y=0^{\prime \prime}$ and P.A. $=7.9$ (Fig. 2). Although the $Y=0^{\prime \prime}$ slit was obtained at different P.A., this slit constrains only the zero point (i.e., systemic) velocity, and any slit passing through the center can be used. This gives $\Omega_{p} \sin i=5.56 \pm 1.39 \mathrm{~km} \mathrm{~s}^{-1} \operatorname{arcsec}^{-1}(70.8 \pm$ $17.7 \mathrm{~km} \mathrm{~s}^{-1} \mathrm{kpc}^{-1}$ ).

We used the FCQ method to measure the line-of-sight velocity curve and velocity dispersion profile of the stellar component along the major axis (Fig. 3). Our measurements at P.A. $=6.9^{\circ}$ are in agreement within errors with those by Simien \& Prugniel (2002) at P.A. $=6^{\circ}$. We derived the circular velocity in the disk region, $V_{c}=94 \pm 19 \mathrm{~km} \mathrm{~s}^{-1}$, after a standard correction for the asymmetric drift as in ADC03. This value is in agreement with the circular velocity derived from the Tully-Fisher relation calculated in the $R$-band by Courteau (1997). Thus, the corotation radius of the bar is $D_{L}=$ $V_{c} / \Omega_{p}=12.7_{-2.9}^{+4.3}$ arcsec and the ratio of the corotation radius to the bar semimajor axis $\mathcal{R} \equiv D_{L} / a_{B}=0.6_{-0.4}^{+1.2}$. The error intervals on $D_{L}$ and $\mathcal{R}$ are $99 \%$ confidence level and were measured with Monte Carlo simulations as in ADC03. By excluding the possibility that $D_{L} / a_{B}<1.0$ as this is thought to be unphysical (Contopoulos 1980), we estimated that the probability that the bar ends close to its corotation radius (i.e., $1.0 \leq$ $\left.D_{L} / a_{B} \leq 1.4\right)$ is $72 \%$. This was calculated via Monte Carlo simulations by assuming a uniform distribution of $a_{B}$ and $V_{c}$ and Gaussian distribution of $\Omega_{p}$ within their error ranges.

\section{DISCUSSION}

By measuring the bar pattern speed of NGC 4431, we have demonstrated that the TW84 method is feasible using stars as a tracer for dwarf galaxies. So far, this technique has been applied only to bright barred galaxies with the result that all the measured bars are consistent with being rapidly rotating (see Corsini 2004 for a review). For NGC 4431 we find a bar suggestive of being fast at $72 \%$ probability, although the measurement uncertainties in this galaxy preclude a stronger statement.

As discussed by Debattista (2003) and Debattista \& Williams (2004), the TW84 measurement of $\Omega_{p}$ is particularly sensitive

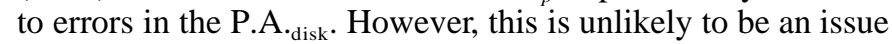
for NGC 4431. In fact, the values of $\mathcal{X}$ and $\mathcal{V}$ measured in the 
offset position at $Y=+5.0^{\prime \prime}$ from the spectrum at P.A. $=$ $7.9^{\circ}$ are in agreement within the errors with those from the two spectra at P.A. $=6.9^{\circ}$. So, the fact that the value of the bar speed parameter of NGC 4431 is nominally less than unity cannot be attributed to the uncertainty on P.A. disk $_{\text {but }}$ bu the scatter in the values of $\mathcal{X}$ and $\mathcal{V}$.

A fast bar in NGC 4431 would suggest a common formation mechanism of the bar in both bright and dwarf galaxies. If disks were previously stabilized by massive DM halos, we exclude that these bars were produced by tidal interactions because they would be slowly rotating (Noguchi 1999). But this is not the case even for galaxies like NGC 4431 and the SB0 NGC 1023 (Debattista et al. 2002), which show signs of weak interaction with a close companion, without being significantly perturbed. This finding allows us to conclude that there is no difference between TW84 measurements in isolated or mildly interacting barred galaxies (ADC03; Corsini et al. 2003).

According to high-resolution $N$-body simulations of bars in cosmologically motivated, CDM halos (see Debattista 2006 for a review), a fast bar would imply that NGC 4431 has a massive disk and does not reside inside a centrally concentrated DM halo. This is in agreement with other studies of dwarf galaxies (Swaters 1999; de Blok et al. 2001; McGaugh et al. 2001; de Blok \& Bosma 2002; Marchesini et al. 2002; Swaters et al. 2003; Spekkens et al. 2005), who analyzed the ionized-gas rotation curves of up to $\sim 200$ objects. By assuming minimal disks and spherical symmetry, most of the data are better de- scribed by DM halos with an inner density core rather than the cusp predicted by CDM.

However, there is no consensus whether these results based on gas dynamics represent an actual problem for the CDM paradigm or whether they could be reconciled with it by taking a variety of observational uncertainties (e.g., slit positioning, spatial resolution), analysis problems (e.g., binning and folding of the rotation curves), and modeling assumptions (e.g., noncircular and off-planar gas motions) into account (de Blok et al. 2003; Swaters et al. 2003; Spekkens et al. 2005). Therefore, we argue that the ongoing debate will benefit from stellar dynamics to constrain the inner mass distribution of DM with the direct measurement of $\Omega_{p}$ in a sample of dwarf barred galaxies by means of integral-field spectroscopy.

E. M. C. and A. P. receive support from grant PRIN2005/32 by Istituto Nazionale di Astrofisica (INAF) and from grant CPDA068415/06 by Padua University. JALA has been funded by the Spanish DGES, grant AYA-2004-08260-C03-01. V. P. D. is supported by a Brooks Prize Fellowship in Astrophysics at the University of Washington and receives partial support from NSF ITR grant PHY-0205413. F. D. B. acknowledges support from the LTSA grant NAG5-13063 and from the NSF grant AST 0607748. V. P. D. and E. M. C. acknowledge the Instituto de Astrofísica de Canarias for hospitality while this Letter was in progress.

\section{REFERENCES}

Aguerri, J. A. L., Debattista, V. P., \& Corsini, E. M. 2003, MNRAS, 338, 465 (ADC03)

Aguerri, J. A. L., Muñoz-Tuñón C., Varela, A. M., \& Prieto, M. 2000, A\&A, 361,841

Athanassoula, E. 2003, MNRAS, 341, 1179

Barazza, F. D., Binggeli, B., \& Jerjen, H. 2002, A\&A, 391, 823 2003, A\&A, 407, 121

Bender, R. 1990, A\&A, 229, 441

Binggeli, B., Sandage, A., \& Tammann, G. A. 1985, AJ, 90, 1681

Bosma, A. 1999, in ASP Conf. Ser. 182, Galaxy Dynamics, ed. D. Merritt, J. A. Sellwood, \& M. Valluri (San Francisco: ASP), 339

Bureau, M., Freeman, K. C., Pfitzner, D. W., \& Meurer, G. R. 1999, AJ, 118, 2158

Contopoulos, G. 1980, A\&A, 81, 198

Corsini, E. M. 2004, in Baryons in Dark Matter Halos, ed. R. Dettmar, U. Klein, \& P. Salucci (Trieste: SISSA), 49.1

Corsini, E. M., Debattista, V. P., \& Aguerri, J. A. L. 2003, ApJ, 599, L29

Corsini, E. M., et al. 1999, A\&A, 342, 671

Courteau, S. 1997, AJ, 114, 2402

Debattista, V. P. 2003, MNRAS, 342, 1194

. 2006, in ASP Conf. Ser. 352, New Horizons in Astronomy, ed. S. Kannappan et al. (San Francisco: ASP), 161

Debattista, V. P., Corsini, E. M., \& Aguerri, J. A. L. 2002, MNRAS, 332, 65

Debattista, V. P., \& Sellwood, J. A. 1998, ApJ, 493, L5 . 2000, ApJ, 543, 704

Debattista, V. P., \& Williams, T. B. 2004, ApJ, 605, 714

de Blok, W. J. G., \& Bosma, A. 2002, A\&A, 385, 816

de Blok, W J. G., Bosma, A., \& McGaugh, S. 2003, MNRAS, 340, 657

de Blok, W. J. G., McGaugh, S., \& Rubin, V. C. 2001, AJ, 122, 2396

de Vaucouleurs, G., de Vaucouleurs, A., Corwin, Jr., H. G., Buta, R. J., Paturel, G., \& Fouquè, P. 1991, Third Reference Catalogue of Bright Galaxies (New York: Springer) (RC3)

Eskridge, P., et al. 2000, AJ, 119, 536
Gentile, G., Burkert, A., Salucci, P., Klein, U., \& Walter, F. 2005, ApJ, 634, L145

Hayashi, E., \& Navarro, J. F. 2006, MNRAS, 373, 1117

Jerjen, H., Binggeli, B., \& Barazza, F. D. 2004, AJ, 127, 771

Knapen, J. H., Shlosman, I., \& Peletier, R. F. 2000, ApJ, 529, 93

Marchesini, D., D’Onghia, E., Chincarini, G., Firmani, C., Conconi, P., Molinari, E., \& Zacchei, A. 2002, ApJ, 575, 801

Marinova, I., \& Jogee, S. 2007, ApJ, in press (astro-ph/0608039)

McGaugh, S., Rubin, V. C., \& de Blok, W. J. G. 2001, AJ, 122, 2381

Moore, B., Governato, F., Quinn, T., Stadel, J., \& Lake, G. 1998, ApJ, 499, L5

Moore, B., Quinn, T., Governato, F., Stadel, J., \& Lake, G. 1999, MNRAS, 310,1147

Navarro, J. F., Frenk, C. S., \& White, S. D. M. 1996, ApJ, 462, 563 1997, ApJ, 490, 493

Noguchi, M. 1999, ApJ, 514, 77

O'Neill, J. K., \& Dubinski, J. 2003, MNRAS, 346, 251

Pedraz, S., Gorgas, J., Cardiel, N., Sánchez-Blázquez, P., \& Guzmán, R. 2002, MNRAS, 332, L59

Prieto, M., Aguerri, J. A. L., Varela, A. M., \& Muñoz-Tuñón, C. 2001, A\&A, 367,405

Sellwood, J. A., \& Debattista, V. P. 2006, ApJ, 639, 868

Simien, F., \& Prugniel, P. 2002, A\&A, 384, 371

Spekkens, K., Giovanelli, R., \& Haynes, M. P. H. 2005, AJ, 129, 2119

Swaters, R. A. 1999, Ph.D. thesis, Univ. Groningen

Swaters, R. A., Madore, B. F., van den Bosch, F. C., \& Balcells, M. 2003, ApJ, 583, 732

Tremaine, S., \& Weinberg, M. D. 1984, ApJ, 282, L5 (TW84)

Valenzuela, O., Rhee, G., Klypin, A., Governato, F., Stinson, G., Quinn, T., \& Wadsley, J. 2007, ApJ, 657, 773

van den Bosch, F. C., \& Swaters, R. A. 2001, MNRAS, 325, 1017

Weinberg, M. D. 1985, MNRAS, 213, 451 